\title{
Filigrane
}

Écoutes psychothérapiques

\section{La lecture-écriture du corps}

\section{Villemaire Paquin}

Volume 18, numéro 1, printemps 2009

Le corps. Sur le divan. Dans le fauteuil I

URI : https://id.erudit.org/iderudit/037720ar

DOI : https://doi.org/10.7202/037720ar

Aller au sommaire du numéro

Éditeur(s)

Revue Santé mentale au Québec

ISSN

1192-1412 (imprimé)

1911-4656 (numérique)

Découvrir la revue

Citer cet article

Paquin, V. (2009). La lecture-écriture du corps. Filigrane, 18(1), 48-59.

https://doi.org/10.7202/037720ar
Résumé de l'article

À partir de 1920, une impasse clinique et théorique confronte la psychanalyse. Elle amène Freud à redéfinir l'inconscient et à élaborer la deuxième topique, poursuivant ainsi la voie de la représentation. Reich, de son côté, en 1934, court-circuite l'appareil psychique et centre sa démarche sur l'énergie, ouvrant la voie du corps. L'impasse, dans un cas comme dans l'autre, ne sera pas pour autant levée dans l'immédiat. Deux vignettes, reliées par l'expression « lecture du corps ", présentent un parcours sous l'éclairage de la psychanalyse contemporaine, du corps écrit, objet d'une lecture au corps lecteur sujet de la lecture. 


\title{
La lecture-écriture du corps
}

\author{
villemaire paquin
}

À partir de 1920, une impasse clinique et théorique confronte la psychanalyse. Elle amène Freud à redéfinir l'inconscient et à élaborer la deuxième topique, poursuivant ainsi la voie de la représentation. Reich, de son côté, en 1934, court-circuite l'appareil psychique et centre sa démarche sur l'énergie, ouvrant la voie du corps. L'impasse, dans un cas comme dans l'autre, ne sera pas pour autant levée dans l'immédiat. Deux vignettes, reliées par l'expression «lecture du corps», présentent un parcours sous l'éclairage de la psychanalyse contemporaine, du corps écrit, objet d'une lecture au corps lecteur sujet de la lecture.

\begin{abstract}
«Les rides et les plis du visage sont les enregistrements des grandes passions, des vices, des savoirs qui se sont exprimés en nous mais nous les maîtres, nous étions absents. » (Benjamin, W., 1971)

«Du point de vue ontologique d'abord, le corps est l'origine et le lieu où la vie se révèle à elle-même. La vie, ici, est entendue comme subjectivité absolue, en tant qu'elle est éprouvée par le sujet qui la vit. Pour le psychanalyste, la vie est affectivité, sentiment de réplétion subjective.» (Dejours, C., 2001,153)
\end{abstract}

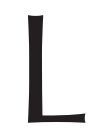

es aléas de la vie m'ont amené à bénéficier, aux plans personnel et professionnel, d'approches thérapeutiques corporelles et verbales situées hors du champ analytique. Étonnamment, c'est leur fréquentation qui me mit d'abord en contact avec la psychanalyse que je côtoie maintenant depuis de nombreuses années. Les fondateurs de ces thérapies, qu'il s'agisse de Reich avec l'analyse caractérielle et l'orgonothérapie, de Lowen avec la bioénergie, ou de Perls avec la Gestalt, étaient tous liées à la psychanalyse soit par leur formation, soit par des emprunts conceptuels au corpus analytique ou encore par leur désir de s'en dissocier. C'est à travers ce parcours où tour à tour, patient, clinicien, théoricien, en moi se croisent et se fécondent que je tenterai de retracer jusqu'à ce jour, le sens qu'a pris pour moi, peu à peu, l'expression lecture-écriture du corps.

Il y a en effet un moment où lecture et écriture sont inséparables, où ce qui se donne à lire s'écrit, au cours du processus. L'étape où soi et l'autre sont indiscernables, dans cette saison de l'archaïque telle que l'appréhende Green, instant où le désir, son objet et le moi se confondent, occasion de création, du trouvé/créé, du lu/écrit. Ce lieu originaire de lecture-écriture, où coexistent des réalités encore indifférenciées, j'aimerais ici le circonscrire. J'emprunterai deux parcours. L'un historique retrace les trajectoires de Reich et de Lowen, la voie du corps. L'autre clinique propose une élaboration métapsychologique à partir de deux vignettes illustrant certaines manifestations du processus de lecture-écriture dérivant d'un corps à corps, la voie de la représentation. 


\title{
Lecture du corps et thérapie corporelle, un angle de vue
}

\author{
«Que le monde soit à lire signifie brutalement qu'un Autre, de l'autre côté, écrit \\ des choses données et qu'avec un bon angle de vue, en principe je pourrais le \\ décrypter. »
}

(Lyotard J.F., 2002, 10)

Nous savons que les traumatismes de guerre et les cas limites (comme celui de l'homme aux loups) ont mis à mal les théories freudiennes basées sur la remémoration et forcé le tournant de 1920. Freud élabore alors la deuxième topique dont l'arrimage avec la première est laissé en plan.

\section{Reich de la psyché vers le corps}

C'est dans ce contexte que Reich tente, à sa façon, de répondre aux défis que rencontrent la clinique, la méthode et la théorie. C'est aux travaux de W. Reich et son analyse caractérielle, élaborée en 1924, puis mise en application entre 1925 et 1933, que l'on songe d'emblée. Sa thèse, reconnue par Freud, présente à l'époque une avancée certaine en ce qu'elle éclaire l'en deçà de la névrose qui met en échec la méthode et la théorie elle-même.

Dès le congrès de 1934, Reich prend position. Sa nouvelle orientation est fonctionnaliste, centrée sur le réflexe orgastique et l'économie sexuelle. Il déclare obsolètes ses conclusions de 1924 tout en maintenant leur validité dans le cadre de la psychologie des profondeurs. W. Reich constate l'impasse dans laquelle se trouve l'analyse face aux patients qui ne peuvent associer librement. Il théorise l'existence de défenses caractérielles dans toute organisation psychique bloquant la libre association de même que le caractère relatif du transfert «dit positif» qui ne peut jouer son rôle de levier dans ces cas (Reich, W., 1972, 178.) Il délaisse donc l'association libre comme règle de base et sa place au sein de la méthode et de la théorie, car elle ne rend pas compte de la réalité clinique. Ainsi, si l'inconscient doit devenir conscient, on doit procéder, du point de vue dynamique, à l'analyse des résistances. Or celles-ci étant structurées (le caractère), il importe également de procéder, du point de vue économique, à l'analyse de cette structure propre à chaque individu. On ne peut donc opérer en s'appuyant uniquement sur la libre association. Il établit ainsi qu'il y a, à la base de l'organisation psychique de chaque individu, une «armure narcissique», hypothèse qui met en doute la possibilité d'un réel transfert positif et remet en question l'idée du transfert comme levier thérapeutique. La formation du caractère, pressentie en 1924 comme la résultante des relations mère-enfant, est théorisée dès lors comme le résultat de la liaison captive de l'énergie végétative et il affirme qu'armure caractérielle et armure musculaire sont identiques. Le travail clinique doit alors libérer, réactiver (stase) cette énergie biologique, propre à tout enfant ${ }^{1}$. L'établissement ou le rétablissement de la fonction orgasmique à travers le réflexe orgastique devient sa «voie royale», la voie du corps, donnant accès à la santé émotionnelle, c'est- 
à-dire, la libre circulation de l'énergie sexuelle (Reich, 1972, 286). Le sexuel se confond ici avec la sexualité. Le caractère signifie dorénavant un comportement bio physique typique et les émotions sont la manifestation tangible de l'énergie biologique de l'organisme qu'il nomme orgone. Dans ce nouveau cadre, ses conclusions de 1924 sont effectivement obsolètes. À partir de 1939, Reich délaisse peu à peu l'analyse caractérielle et poursuit, jusqu'à sa mort, ses tentatives visant à isoler cette énergie. Il croit réussir grâce à l'accumulateur d'orgone. Il tente de suppléer, nous semble-t-il, à un déficit énergétique du psychisme à la manière d'un supplément d'hormone de synthèse pour un appareil hormonal déficient.

\section{Lowen du corps vers la psyché}

Alexander Lowen, un élève et ex-patient de Reich, inconfortable avec les dernières théories (orgone) et les positions, à son avis, sectaires de ses adeptes, reprend à son compte l'analyse caractérielle et pousse plus avant les interventions directes sur le corps, démarche amorcée par Reich, pour favoriser la fluidité de l'énergie. Il développe une méthode qu'il nomme la «bioénergie ». Dans Bioenergetics: La thérapie révolutionnaire qui utilise le langage du corps afin de guérir les problèmes de l'esprit publié en 1975, A. Lowen prétend que le bioénergéticien peut, à la lecture du corps d'une personne, retracer l'histoire de sa vie comme un forestier peut le faire en examinant les anneaux de croissance d'un arbre (Lowen, 1975, 57).

Lowen rapporte, dans cet ouvrage, le travail thérapeutique réalisé avec Reich au début des années 1940. L'établissement du réflexe orgastique, critère fonctionnel de la santé émotionnelle selon Reich à cette époque, en est le centre. Lowen n'y cache pas son admiration pour Reich et ses efforts pour répondre au maître. Malgré cela, de l'avis de Lowen lui-même, la réussite de sa thérapie est partielle, car, si le réflexe orgastique est remis en route, il n'est pas toujours au rendez-vous et la satisfaction sexuelle laisse à désirer, malgré l'atteinte de l'objectif thérapeutique. L'impasse n'est pas levée pour autant. D'un commun accord ils décident de suspendre les séances. Il quitte les États-Unis pour faire des études de médecine en Suisse. De retour au pays après quelques années, Lowen veut reprendre sa démarche thérapeutique interrompue, mais Reich s'est entre temps centré sur le développement de son accumulateur d'orgone et n'est plus disponible. C'est donc avec un collègue de formation reichienne première génération (végéto-thérapie) qu'il entreprend cette thérapie à deux mains, pourrait-on dire puisque son collègue, Pierrakos, s'occupe de dissoudre les tensions musculaires par des manipulations, pressions et massages, alors que Lowen expérimente sur lui-même des exercices de mise en charge, l'accumulation naturelle d'énergie. Les tensions ou blocages, ainsi mis en évidence, Pierrakos tente de les dénouer par la suite. Un arsenal d'exercices est ainsi mis au point et testé sur lui-même.

Au cours de cette démarche, il s'éloigne de l'établissement du réflexe orgastique comme unique objectif du travail physique au profit de la dissolution des tensions qu'il met en lien avec les problèmes de personnalité. L'analyse 
caractérielle de Reich redevient alors, un élément majeur de sa théorisation, toutefois elle a perdu, depuis 1930, sa dimension psychanalytique : la question de l'inconscient est évacuée bien que la remémoration (première topique) demeure un objectif thérapeutique essentiel. La théorisation est davantage phénoménologique.

«Si le passé est exclu, le futur n'existe pas. »(Lowen, 1975, 34)

«J'ai alors conclu qu'aussi importante que soit l'emphase sur le travail des tensions musculaires, l'analyse de la façon de faire habituelle de la personne et ses comportements méritaient une égale attention. » (Lowen, 1975, 42)

Dans son œuvre, l'expression « thorough analysis » m’a longtemps intrigué. Je l'associais confusément à la psychanalyse pendant mes années de formation (1980-1985). Elle se réduit finalement à l'analyse des comportements conscients. Toutefois, l'irruption d'affects et leur intensité, accompagnant l'émergence des souvenirs enfouis, était reconnue comme condition de libération et d'intégration (Lowen, 1975, 62). Dans ses relations thérapeutiques avec Reich ou Pierrakos, il ne sera pas question du transfert ou de son utilisation comme levier. Lowen n'est pas à théoriser le rapport à l'autre comme facteur constituant de la personne. Il est dans un intrasubjectif sans appareil psychique comme tel. S'il insiste sur la remémoration, c'est la libération de l'énergie qui demeure l'objectif, énergie liée au passé, captive dans le corps dont la remémoration signe la libération.

\section{Lowen, le corps et l'esprit}

«Ma position est que les processus énergétiques du corps déterminent ce qui
advient dans l'esprit ${ }^{2}$, de la même façon qu'ils déterminent ce qui se passe dans
le corps.» (Lowen, 1975,44 )

Lowen laisse dans l'indétermination la manière dont les processus énergétiques opèrent pour déterminer ce qui advient dans l'esprit et le corps (on pense à la pulsion et à la représentation). Cette position ressemble à la «boîte noire » du groupe de Palo Alto ${ }^{3}$. Technicien plus que théoricien, il est un de ceux qui ont vulgarisé l'expression «langage du corps » et mis la «Nature humaine », on pense à l'homme de Rousseau, au panthéon des dieux laïcs des années 1960. La Nature sait, organise. Il s'agit alors en substance de lever les barrières qui empêchent la Nature d'exercer son projet dans le corps, par la libre circulation de l'énergie. Pour parler comme Lyotard, il s'agit là d'un angle de vue. Cela dit, le corps et le travail corporel constituent, pour de nombreux patients, une porte d'entrée non négligeable, bien concrète, à l'intérieur d'eux-mêmes, une étape souvent incontournable, une première rencontre avec quelque chose de soi, en soi, qui se présente en mal d'être représenté. Le travail physique sensibilise par la mise en évidence des sensations, des mouvements corporels involontaires, équivalents des 
comportements sociaux spontanés, à l'existence de ce quelque chose en soi dont le visible des actes physiques, émotifs, comportementaux sont une traduction ou transduction comme le suggère $\mathrm{D}$. Scarfone.

Comment s'inscrivent les tensions dont parle Lowen? Sur les plans théorique et clinique, Lowen comme Reich occultent les processus d'inscription comme tels et l'émergence d'une parole qui leur serait liée, comme si la connaissance de l'alphabet suffisait pour inspirer la parole du poète en l'homme.

\title{
Non seulement le corps, mais aussi la psyché
}

\author{
«Là où un homme rêve et délire, un autre se lève qui interprète; ce qui était déjà \\ discours, même incohérent, rentre dans le discours cohérent par l'hermé- \\ neutique. » (Hegel cité par Lyotard J. F. 2002, 12)
}

\section{Rolfing, seulement le corps?}

J'aimerais maintenant, par le biais d'une première vignette issue d'une expérience personnelle, présenter une intervention directe visant une restructuration du corps en fonction d'un modèle de référence: l'intégration structurale d'Ida Rolf (rolfing). Cette approche postule qu'une action sur la structure corporelle est une action sur la personnalité, qu'une structure corporelle équilibrée est le pendant d'une personnalité équilibrée et donc l'équilibre structural corporel est la référence visible de l'objectif à atteindre. Je proposerai, par la suite, un essai de compréhension métapsychologique de ce qui s'y passe.

\section{a) La levée de la barrière à l'arraché}

Le coude bien crémé, le thérapeute pèse de tout son poids sur les muscles de la cuisse en vue d'assouplir la gaine aponévrotique permettant son expansion et sa souplesse. Un gémissement accompagne, pourrait-on penser, la douleur; or celuici s'enfle en un sanglot qui devient incontrôlable. Le «rolfeur» (le praticien de cette approche) cesse sa manipulation, le sanglot persiste un certain temps, s'atténue peu à peu, s'arrête. Le praticien poursuit son travail, silencieux.

«As he moves toward structural balance, he knows that his psychological make-up has changed as well. He can experience to his satisfaction that his psychological hang-ups are literal thorns in literal flesh. They can disappear only as the flesh changes, as the barriers within the flesh are disengaged, and as the free flow of body energy and fluids is established [...] change in the flesh must occur in order for there to be a successful therapeutic outcome. » (Rolf, 1977, 18) ${ }^{4}$

Revenons à la vignette où le coude force l'assouplissement de la gaine entourant les muscles de la cuisse et donne plus d'espace à la chair du muscle. Une 
énigme se fait jour: les sanglots; va pour des pleurs accompagnant une douleur, mais les sanglots? Nous savons que la glande lacrymale s'active pour éliminer un grain de poussière, un trop de douleur, de joie, de tristesse à voir, à vivre, comme si la larme protégeait l'œil autant du feu de l'irritation physique que du feu de l'émotion. Nous savons aussi que le sanglot ajoute la participation du viscéral et implique un relâchement de la musculature abdominale, le contenant des viscères. Ainsi, du fond du bassin, de l'anus à la bouche, une voie est ouverte, donnant à la voix une caisse de résonance, participation du viscérale, soubresauts de tout le corps. Le corps entier pleure sans retenue, sans barrière, par le simple exercice d'une pression sur la cuisse! Qu'en est-il?

Le sanglot et la cuisse, le sanglot dans la cuisse, la barrière dans la cuisse ? Cuisse de Jupiter matrice de la naissance d'Athéna protectrice d'Athènes. Rester debout... résister, tomber à genoux... concéder, mettre à genoux... vaincre l'adversaire. Voilà plusieurs expressions qui consacrent un lien entre une position physique (plier ou non le genou) et des sentiments contraires: la soumission, la résistance, la victoire, la défaite ou encore, ultimement, la dignité, mourir debout une «manière de faire non» (R. Roussillon, 2008,103). Situation limite à reconnaître si on veut vivre, à nier si on ne peut que survivre lorsque soumission et dépendance s'équivalent. La nécessaire prise en compte de la réalité de la dépendance devient inconciliable avec être, omnipotence oblige. Dans la mythologie grecque, le héros ne peut qu'appeler la mort de ses vœux pour éviter la soumission, un déshonneur. Tout, mais pas ça. Le samouraï lui aussi, se fait «hara-kiri» plutôt que d'être sans maître à servir sens de sa «vie». Sous la protection de la cuisse de Zeus, le muscle des muscles du dieu des dieux, géniteur d'Athéna, Athènes ne court pas le risque de la soumission, elle ne pliera pas le genou, avec de telles cuisses.

\section{b) L'inconscient comme propulseur de la forme}

Freud, à plusieurs reprises, prend à témoin dictons populaires et légendes pour soutenir la validité d'un questionnement, si ce n'est d'une hypothèse, d'une idée, sans en poursuivre ultérieurement l'élaboration. L'une d'elles concerne la plastique comme attribut de l'inconscient. Dans sa réponse à Groddeck, réclamant un élargissement du champ d'action de l'inconscient pour y inclure le somatique, Freud définit la cinquième caractéristique de l'inconscient comme «l'action plastique ${ }^{5}$ intense que n' obtient jamais l'acte conscient » (Freud S., 1966, 345).

Françoise Coblence, dans son livre Les attraits $d u$ visible, définit cette action comme: une «puissance créatrice de formes» affectant les corps: organique, hystérique, sensible dont les sensations relaient, à la conscience court-circuitée, l'information que quelque chose s'est passée, signet de l'action plastique. Dans ce cadre l'empathie est alors présentée comme une des formes de cette puissance. Elle serait "“imitation esthétique" de l'autre»(Coblence, 2005, 101 à 103). Le propos de Coblence sous-tend plusieurs réalités d'importance pour notre compréhension de la problématique de la «lecture-écriture du corps». Nous sommes dans un espace entre deux postes frontières théoriques, le physique et le psychique. Afin 
de poursuivre, nous proposons une passerelle entre le concept de la puissance créatrice de forme et le principe de "convergence-cohérence ${ }^{6} »$ des Botella à la base du travail de figurabilité.

Nous supposons qu' un principe similaire, sinon identique balise la «puissance créatrice de formes». Tentons de le voir au travail dans la mythologie, les concepts et la vignette. Ainsi la narration de la naissance d'Athéna, est une démonstration du principe à l'œuvre à travers la forme mythologique en ce qu'elle tend à faire converger toutes les données du moment, en une intelligibilité mythologique d'une représentation de la protection face à une menace; au plan des concepts, «l'imitation esthétique» est vue comme une forme de la répétition, au service de cette puissance, comme dans l'expression: se tenir debout sans relâche puis une forme de la relation à l'autre, à travers l'imitation, comme le héros, et finalement, le moteur sinon le désir qui se manifeste par une sensation, «sensation relais » nous dit Coblence, de la résistance puis du relâchement, prélude aux sanglots dans la vignette. Nous comprenons cette «sensation relais» comme quelque chose qui signe une mise en lien. L'intérêt d'une juxtaposition des points de vue des Botella et de Coblence tient aussi dans ce que l'une (dans ses termes) est plus près du visible et l'autre de la représentation alors que toutes deux s'intéressent au même processus, au passage des frontières entre le corps et la psyché.

Dernière pièce à mettre en place: une conception de l'appareil psychique qui rend possible le passage des frontières du physique au psychique. Pour rendre compte de ce travail psychique dès l'aube de notre existence psychique nous pensons à A. Green répondant à J. Kristeva au sujet de l'appareil psychique et son dualisme avec l'appareil de langage: «Par "appareil psychique" j'entends quelque chose qui va bien au-delà de l'affect, aussi bien des mouvements corporels, des formes de communication gestuelle ou autre, etc.» (Green, 2005, 392)

L'activité de l'appareil psychique ainsi pressentie prend en compte l'en deçà de la représentation et sa nécessaire élaboration. Nous sommes donc à l'aurore du signifiant, à l'orée de l'insignifiant, espace d'un «protolangage» dont le corps garde les marques, une «lecture-écriture du corps» sensible et ou visible, une première base de représentation, comme un pictogramme (Aulagnier) dans la psyché. Moins l'environnement est modérateur, plus la réponse physique se répète près de l'identique, plus se creuse une empreinte qui alors attire et gomme les différences de sorte qu'à des situations hétérogènes une même réponse est donnée. Cette réaction caractérise-t-elle le corps dans ces formes, un destin de la pulsion ${ }^{7}$ qui n'arrive pas à être psychisée? Est-elle figée dans son expression corporelle, dont la sensation révélerait la présence?

\section{c) Suite et fin de la séance de rolfing}

La mythologie «transduit» l'instinct de protection animale en une représentation: une cuisse matrice. La cuisse de Zeus la force brute, la puissance créatrice de forme, enfante Athéna déesse de la guerre protectrice d'Athènes. La mythologie relate le fruit d'une réflexion, une convergence cohérente, et de ce fait humanise, 
psychise, l'instinct de protection par la représentation et tout en reconnaissant la dépendance, la contrecarre. Nous y lisons une métaphore du processus de lectureécriture du corps.

\section{Cuisse matrice dans la séance}

Le «rolfeur» orthodoxe interrompt sa pression et attend, non-ingérence, nonindifférence que le sanglot s'atténue, se taise, alors il poursuit là où il s'était arrêté. Tout se passe dans la chair, il y a une barrière, nous dit Rolf. Dans cette chair, une épine-barrière a été enlevée, la preuve: le sanglot, le sentiment d'unification et d'intégration qui lui succède. La lecture du corps se limite au plus ou moins d'élasticité du muscle et visuellement à la conformité de la posture au schéma idéal de référence. La réussite de son intervention est en relation avec la pression qu'il peut exercer, son poids, sa force et la résistance de la musculature. Une lecture et un travail pour rétablir une conformité à un modèle postural de référence, l'équilibre structural.

Ce modèle d'intervention postule qu'il y a inscription des tourments psychologiques sous forme de barrières dans la musculature, qu'Ida Rolf appelle globalement la chair, qui sont autant d'épines à extraire. Apparemment, le massage y parvient. Mais comment s'est faite cette inscription ? Pourquoi le massage est-il opérant? C'est la métaphore du plombier qui nous répond: une fois le tuyau débouché, il n'y a plus d'obstruction, l'eau s'écoule librement. L'énergie corporelle et les fluides circulent, l'entrave levée, selon le plan initial. Conçu par qui, par quoi ? En tenant compte de l' «énergie» dont parle l'auteur, il semble implicite qu'elle se réfère, comme Lowen, à la doctrine vitaliste. Elle rend la matière vivante et organisée selon la définition qu'en donne le Petit Robert (2003); toutefois, ce principe est admis au plan psychologique sans qu'elle en précise les modalités de fonctionnement. Dans ce cadre, il est logique que la levée des barrières permette à l'organisation de s'actualiser.

\section{Suite et fin}

Ce que le «rolfeur» n'a jamais su de la part de son patient, la parole n'ayant pas de place dans ce modèle, c'est qu'à la suite de cette séance il a produit un dessin. Sa particularité est de présenter un corps aux muscles saillants, les cuisses en particulier, un corps d'adulte, coiffé d'une tête d'enfant. Impossible, se rappelle le patient, d'esquisser harmonieusement une tête d'adulte. L'un donne à voir la force, l'autre la faiblesse, et l'observateur a sous les yeux un désarroi. Le désarroi n'est pas sur la figure de l'enfant, il est l'affect qui se donne à voir, à ressentir, sans se faire voir comme tel, par l'incohérence du rapport tête corps, tout comme le sanglot se donnait à entendre sans cohérence apparente avec la manipulation, les deux se complètent, comme éléments d'un rébus. On peut alors penser que devant l'incohérence d'une situation provoquant un désarroi, le corps est devenu rigide, résistant, une armure, dirait Reich, en lieu et place d'une capacité de penser à venir, ce dont la tête d'enfant est toujours en attente. 
L'intervention physique, guidée par une lecture du corps, laisse en plan l'élaboration: d'un sens, d'une parole, de la relation entre les protagonistes, en d'autres termes elle laisse de côté le processus d'humanisation, la nature humaine dirait probablement Winnicott. Elle ne nomme pas. La relation entre le massage et la psychologie est postulée. Si nous ouvrons cette relation présumée, nous pouvons concevoir que ce massage induit une sensation, non seulement celle du corps souffrant, — le gémissement — mais aussi, suite au sanglot, une expérience paradoxalement satisfaisante. D'une part, il y a la dissolution de la tension musculaire bien réelle par effraction, en soi douloureuse, et d'autre part (et d'un autre ordre), grâce à l'abréaction obtenue par le sanglot, une baisse de la tension, cette fois-ci émotive, d'où un apaisement, une satisfaction.

\section{La voie du corps}

Nous retenons de cette vignette, pour la suite de notre propos, la dimension d'expérience d'une sensation satisfaisante, en elle-même intéressante à retrouver. Dans l'armure, une brèche de plaisir est créée, une voie pour le désir est ouverte, un domaine d'activité pour le semblable (DeMuzan) est apparu, un levier est disponible. Le dessin transcrit l'impasse en image. La prochaine vignette centrera notre attention sur le «lecteur», l'impact d'une rencontre, l'effet d'une lecture.

\section{Lecture du corps, un exercice}

«Le vrai symbole donne à penser, mais d'abord il se donne à voir et que tout discours s'épuise avant d'en venir à bout. » (Lyotard J.F. 2002, 13)

Une jeune femme dans la trentaine, en maillot deux pièces — lecture du corps oblige - prend une position de tension, celle dite de l'arc. Son poids est déporté légèrement vers l'avant du pied, ceux-ci pointés à peine vers l'intérieur. Les poings exercent une pression sur l'arrière du bassin le maintenant ainsi vers l'avant tout en ouvrant la cage thoracique. La tête est posée dans la continuité de l'arc que forme le corps. Comme lecteur, je me place à deux ou trois mètres devant elle de sorte que j'ai une vue d'ensemble du corps. Je regarde la personne en choisissant un point focal hors foyer.

Une image s'impose rapidement: la tête d'un dragon des fêtes chinoises. Je suis surpris et certes inconfortable devant ce dragon qu'elle est devenue. À qui le dragon, à moi ou à elle, à nous, et que dit-il ? Je n'en sais trop rien. Cette «lecture » actualise en moi un inconfort relié à la présence de la jeune femme. Lecture? Lecture certes et aujourd'hui un souvenir-écran que je vais utiliser pour la suite de mon propos.

Dans Les attraits du visible Françoise Coblence souligne: « ... dans le registre visuel, quantité et qualité, affect et représentation s'articulent dans le rapport à autrui.» (Coblence, 2005, 54). Cette tête de dragon figure donc quelque chose de la qualité de la relation entre les protagonistes du point de vue du lecteur, ce qui ne peut laisser indemne la suite de leur interaction. Une tête de dragon les relie et 
les sépare. On peut dès lors présumer, d'une part, que les associations du lecteur pourraient amorcer une perlaboration menant à penser la situation et, d'autre part, que la relation entre eux est, comme telle, un paramètre de la situation globale, sans chercher à y départager «le tien du mien.»

Pour que puisse avoir lieu cette pensée, il doit exister un rapport affecté à un autrui, permettant l'activité de comparaison que ce soit en soi-même avec soimême ou avec un autre, d'autre part un écart, afin de ne pas être qu'affect. La tête de dragon est l'image sensible de ce rapport à autrui. Je songe également à l'empathie définie par Freud en 1921: «... comme la saisie en l'autre, de ce qui est étranger à mon moi et ce qui est étranger au sien.» (Freud cité par Coblence, F., 2005, 50). Dépliant cette définition, F. Coblence montre qu'il y a nécessairement comparaison et distance suffisante lorsqu'il y a empathie.

Dans le cas présent, la tête de dragon me laissa pantois, en deçà de l'empathie, car sans distance suffisante pour penser quelque chose. L'image $^{8}$ » me soulagea en figurant ma terreur, en paraphrasant les Botella, sans plus. L'énigme d'alors est restée en friche. Aujourd'hui, à l'occasion de cet écrit, vingt-cinq ans plus tard, guidé par leurs travaux sur la figurabilité, il me semble y voir plus clair. Le regard hors focus je le nomme «l'attention flottante visuelle» car il me semble faciliter, tout comme l'«attention flottante» l'«accident de penser», l'accident de perception un «regarder», à l'œuvre lors de cet exercice. Le «regarder», est d'abord une capacité de reproduire ce qui fut ressenti sous le regard de la mère. L'appellation «regarder», (Botella C. et S. 2007, 98) est en quelque sorte un emprunt à son avenir, car en son origine il n'est pas visuel mais de l'ordre du ressentir. La définition de Green de l'archaïque comme un état où le désir son objet et le moi se confondent, trouve ici son application exacte, me semble-t-il. C'est donc l'inconfort dans la relation, ses références inconscientes qui sont colligées condensés dans l'image, elle même matériau à déplier sans fin, comme un rêve.

Cet exercice de lecture dévoile la part subjective inconsciente, à plus ou moins forte dose, inhérente à toute «lecture», même si elle se veut diagnostic objectif, par suite d'un relevé des lieux de tensions musculaires, de la posture corporelle globale, des zones mortes du corps, signalées par l'absence de couleurs, un différentiel de chaleur, une rigidité des mouvements, de la respiration, monnaie courante du bio-énergéticien. Elle est reliée à une saisie inconsciente dont seule la forme de l'affect est présentée comme cette vignette l'illustre, une image. C'est l'occasion, à la croisée du premier regard, lors du premier instant de toute rencontre, pour l'inconnu en soi et de l'autre de se manifester; si toutefois, l'espace interne est suffisant. La pensée s'active, sinon psyché rumine, forçant la mentalisation ou la somatisation. Le corps n'arrête pas de lire, psyché n'arrête pas d'en présenter sa lecture!

Pour qu'il y ait lecture, la rencontre est nécessaire, mais pas suffisante, il faut aussi être en mesure de «regarder», de contenir le ressenti et d'en penser quelque chose dans la relation. Alors, l'image, entendue comme une traduction de la rencontre, la «re-présente», puisque le souvenir de cette image propose à nouveau 
ce qui avait pris forme: une traduction par figuration d'un ressenti d'une relation. Avais-je été devant cette femme-dragon une sorte de Champolion devant la pierre de Rosette, incapable de se rappeler son grec (sa préhistoire), le pont entre les hiéroglyphes et le français? Il est important de souligner avec Françoise Coblence que c'est l'inquiétant de l'inconscient qui sera écarté de la traduction (Coblence, F., 2005, 78). Qu'en est-il quand, les yeux ouverts, le lecteur ne voit rien à traduire alors que dans son corps une lourdeur se donne à ressentir? Nous pensons à ces moments pénibles où la somnolence, véritable tombée de rideau, occulte scène et image, isole l'analyste dans une nuit sans rêverie. Le sommeil lui tombe dessus et c'est le réveil qui le lui révèle sans rien dire de ce qui l'a ensommeillé ou encore, c'est un combat, comme celui de l'automobiliste contre le souhait de fermer les yeux, oh! juste un instant. La solution est si attrayante malgré le mortifère de la proposition, la néantisation de l'analysant, de l'analyste et de l'analyse, la sortie de route. La rencontre devient un corps à corps dans la tranchée où règne la loi de l'un ou l'autre. L' 'impossible» est au rendez-vous il faut le penser.

Conclusion

Nous disions en introduction qu'il y a un moment où ce qui se donne à lire s'écrit. Nous en sommes là. Nous soulignerons en conclusion, avec Scarfone (D, Scarfone, 2006, 1) que la réalité est une question d'investissement. La qualité de cet investissement permet de jeter un pont qui relie le soi, moi-monde unifié, au moi, moi/monde séparé. Malgré le contact sans solution de continuité entre corps et monde environnement, le tablier du pont est le soi bien établi du médiateur (B. Brusset, 2005, 194) et la réalité entre eux des paramètres relationnels. Il constitue un environnement suffisamment bon pour que d'une lecture-écriture adviennent deux sujets, un analysant, un analyste, sans perdre de vue qu'au départ il y a la cire : l'espace corporel.

villemaire paquin 290, empire, greenfield Park qc j4v 1v2. villemairepaquin@hotmail.com

\section{Notes}

1. L'implicite de cette position est le vitalisme: «"force vitale" qui rend la matière vivante et organisée.» Petit Robert

2. J'ai traduit mind par esprit en tenant compte des deux versants: réalité pensante et souffle de vie, ce qui me semble rendre la conception de Lowen.

3. Le groupe de Palo Alto, dans les années soixante, avait décrété que l'inconscient était l'équivalent d'une boîte noire dont on ne saurait connaître le contenu. Je songe aux boîtes noires des avions dont l'analyse sert à déduire les décisions prises avant l'accident, des traces de décisions autrement inconnaissables.

4. «En s'approchant de l'équilibre structural, il sent que son équilibre psychologique a aussi changé. Une satisfaction accompagne la découverte que ses tourments psychologiques sont littéralement des épines incrustées 
dans sa chair. Elles peuvent disparaître seulement après modification de la chair, suite à la levée des barrières sises dans la chair elle-même et l'établissement de la libre circulation de l'énergie et des fluides corporels [...] sans changement dans la chair pas de succès thérapeutique.» (ma traduction, mes italiques)

5. La plastique: «Qui a le pouvoir de donner la forme.» Selon Le Petit Robert.

6. Nous reprenons ici la définition que donnent les auteurs du travail de figurabilité : «Procédé primordial de toute liaison, "l'une des fonctions les plus précoces" (Freud, 1920) de la vie psychique, le travail de figurabilité produisant l'intelligibilité du rêve, est un processus psychique qui, se déroulant sur la voie régrédiente, serait déterminé par la tendance à faire converger toutes les données du moment. Liant stimuli internes et externes présents en une seule unité intelligible, marqué par une simultanéité atemporelle, dont la forme originaire la plus élémentaire serait une figurabilité, une intelligibilité hallucinatoire....le travail de figurabilité représenterait la "voie royale" de toute intelligibilité. » (Botella, C., et S., 2007, 83ss)

7. Nous pensons au modèle pulsionnel deux temps de Winnicott, tel que décrit par Reid 2006, 1547

8. «La "vue" aura la tâche de combler le vide, d'éviter l'état de détresse; "l'image" adviendra pour soulager la terreur en donnant forme à l'affect. » (Botella, C., et S., 2007, 100)

\section{Bibliographie}

Aulagnier, P., 1975, La violence de l'interprétation, Paris, Puf, 1995.

Benjamin, W., 1971, Mythe et violence, Paris, Denoël.

Botella, C., et S., 2007, La figurabilité psychique, Paris, In press.

Botella, S., 2005, Racines traumatiques du complexe d'Edipe, in Richard, F., Uribari, F., Autour de l'œuvre d'André Green, Paris, Puf, 343-359.

Brusset, B., 2005, Psychanalyse du lien, Paris, PUF.

Coblence, F., 2005, Les attraits du visible, Paris, PUF.

Dejours, C., 2001, Le corps d'abord, Paris, Payot.

Freud, S., 1966, 1873-1939 Correspondances, Paris, Gallimard.

Lowen, A., 1975, Bioenergetics, Markham, Penguin Books, 1981.

Lyotard, J., F., 1971, Discours, figure, Paris, Klincksieck.

Reich, W., 1945, Character Analysis, Moon Day Press, New York, 1972.

Reid, W., 2004, Le contre-transfert, la symbolisation et le don d'absence, in Filigrane, n ${ }^{\circ}$, 34-47.

Reid, W., 2006, De l'ensemble individu/environnement à la troisième topique : la pulsion, le narcissisme, l'emprise et la relation d'objet, in Revue française de psychanalyse, $\mathrm{n}^{\circ} 5,1547$.

Reid, W., 2008, Un nouveau regard sur la pulsion le trauma et la méthode analytique. Première partie : une théorie de la psyché..., in Filigrane, $\mathrm{n}^{\circ}$ 1, 34-47.

Roussillon, R.,2008, Le jeu et l'entre-je(u), Paris, PUF.

Scarfone, D., 2006, L'examen de réalité une histoire d'amour, texte inédit. 